\title{
Investigating the Feasibility of Using Mplus in the Estimation of Growth Mixture Models
}

\author{
Ming Li \\ University of Maryland, liming@umd.edu \\ Jeffrey R. Harring \\ University of Maryland, harring@umd.edu \\ George B. Macready \\ University of Maryland, macready@umd.edu
}

Follow this and additional works at: http://digitalcommons.wayne.edu/jmasm

Part of the Applied Statistics Commons, Social and Behavioral Sciences Commons, and the Statistical Theory Commons

\section{Recommended Citation}

Li, Ming; Harring, Jeffrey R.; and Macready, George B. (2014) "Investigating the Feasibility of Using Mplus in the Estimation of Growth Mixture Models," Journal of Modern Applied Statistical Methods: Vol. 13 : Iss. 1 , Article 31.

DOI: $10.22237 /$ jmasm/1398918600

Available at: http://digitalcommons.wayne.edu/jmasm/vol13/iss1/31

This Algorithms and Code is brought to you for free and open access by the Open Access Journals at DigitalCommons@WayneState. It has been accepted for inclusion in Journal of Modern Applied Statistical Methods by an authorized editor of DigitalCommons@WayneState. 


\section{Investigating the Feasibility of Using Mplus in the Estimation of Growth Mixture Models}

\author{
Ming Li \\ University of Maryland \\ College Park, MD
}

\author{
Jeffrey R. Harring \\ University of Maryland \\ College Park, MD
}

\author{
George B. Macready \\ University of Maryland \\ College Park, MD
}

Hipp and Bauer (2006) investigated the issues of singularities and local maximum solutions within growth mixture models (GMMs) and made recommendations regarding the use of multiple starting values. Building on their work, this simulation study investigates the feasibility of estimating GMMs within Mplus as measured by convergence to proper, but local solutions.

Keywords: Local maximum solution, convergence, growth mixture modeling, EM algorithm

\section{Introduction}

There continues to be growing interest in applying finite mixture models to established statistical methods with the primary goal of accounting for population heterogeneity in model parameters where group membership is latent. One such hybrid is growth mixture modeling (GMM; Muthén, 2001; Muthén \& Shedden, 1999) which combines latent growth modeling for the analysis of repeated measures data and latent class analysis (Muthén, 2004). Though GMMs have the advantage of determining possible presence of latent subpopulations with qualitatively distinct patterns of development over time, like many other mixture model applications, GMMs present particular estimation difficulties such as reaching local rather than global optima and, in the case of mixtures of normal distributions, singularities on the likelihood surface (see, e.g., Böhning, 1999). Estimation algorithms for GMMs will impact the convergence rate to proper, global solutions as alternative strategies will likely interact differently with the likelihood surface. Direct maximization of the loglikelihood using gradient

Ming Li is a fifth yearPhD candidate. Email at: liming@umd.edu.Dr.JeffreyR. Harring is Associate Professor. Email at: harring@umd.edu. Dr. George B. Macready is Professor. Email at: macready@umd.edu. All are part of Measurement, Statistics and Evaluation in the Department of Human Development and Quantitative Methodology. 


\section{ET AL}

methods like Newton-Raphson although cumbersome, can be very efficient especially if the intermediate solution is near the maximum (Hsu, 2011). The expectation-maximization (EM) algorithm (Dempster, Laird \& Rubin, 1977), on the other hand, provides an indirect approach to obtain maximum likelihood (ML) estimates and is well-suited for estimating GMMs (Muthén \& Shedden, 1999). The EM algorithm is an iterative optimization strategy motivated by configuring the statistical model or method as a missing data problem by considering the conditional distribution of what is missing given what has been observed. However, a known deficit of the algorithm is its relatively slow speed to converge (or lack thereof). Yet, the popularity and usefulness of the EM algorithm for GMM applications stems from its seemingly simple implementation and how reliably it can ascertain global optima through stable, uphill steps. This is the primary estimation strategy used in Mplus.

The preponderance of methodological studies investigating GMMs has focused on correct class enumeration and parameter recovery across a variety conditions thought to directly influence the hypothesized mixture of latent growth models. To discriminate between local and global solutions and, in general, to avoid likelihood surface irregularities it has been recommended that multiple sets of starting values be used when estimating parameters for finite mixture models (McLachlan \& Peel, 2000; Muthén, 2001). The question arises then of how these starting values should be chosen so that they satisfactorily span the parameter space (Hipp \& Bauer, 2006) and at the same time do not skirt too close to its space boundaries where divergence is more likely to occur (McLachlan \& Basford, 1988). The default in Mplus is to generate 10 random sets of starting values although a number of recent studies have encouraged increasing this number in the face of greater model complexity and minimal class separation (Hamilton, 2009; Kohli, 2010; Tolvanen, 2008). In general, these studies have utilized maximum likelihood estimation vis-à-vis the EM algorithm to fit a particular growth mixture model using the mixture modeling module in Mplus. However, only the study by Hipp and Bauer (2006) has attempted to qualify the conditions under which estimation of GMMs fails in terms of computational machinery in this modeling and software context.

Building on this work, the primary objectives of this research project are: (1) to empirically investigate the feasibility of the estimation of GMMs within Mplus as measured by convergence to a proper, global solution under increasing model complexity and realistic data analytic conditions; and (2) to provide recommendations to practitioners as to what can be expected from the algorithm when applying these models in practical research settings. Issues are examined 


\section{MPLUS IN GMM ESTIMATION}

related to combating local solutions and nonconvergence including quality of starting values, random perturbations of those values, the number of sets of those starting values, and manipulated arguments in the mixture module related to the EM algorithm on the fitting of GMMs under increased model complexity.

\section{Methodology}

\section{GMM Specification}

The standard latent growth model can be written as

$$
\mathbf{y}_{i}=\Lambda \boldsymbol{\eta}_{i}+\boldsymbol{\varepsilon}_{i}
$$

where $\mathbf{y}_{i}$ is a $p \times 1$ vector of observed continuous repeated measures for individual $i$, where $p$ denotes the number of waves of data, $\boldsymbol{\eta}_{i}$ is a $q \times 1$ vector of latent growth factors defining the trajectory where $q$ is the number of latent growth factors ( $q=2$ for a linear trajectory with intercept and slope), and where $\boldsymbol{\varepsilon}_{i}$ is a $p \times 1$ vector of time-specific residuals for individual $i$, and is typically assumed to be distributed normally, $\boldsymbol{\varepsilon}_{i} \sim N_{p}\left(\mathbf{0}, \Theta_{i}\right)$. The functional form of the individual trajectories is defined by basis functions (columns of $\boldsymbol{\Lambda}$ ) whose elements may be constants or parameters to be estimated. For a linear trajectory with latent intercept and slope factors for $p$ equally spaced repeated measures, $\boldsymbol{\Lambda}$ would be set to $\boldsymbol{\Lambda}=(\mathbf{1}, \mathbf{t})$, where $\mathbf{1}$ is a $p$-dimension vector of ones and $\mathbf{t}=(0,1, \ldots, p-1)^{\prime}$.

The joint density of $\boldsymbol{\varepsilon}_{i}$ and $\boldsymbol{\eta}_{i}$ is assumed to be multivariate normally distributed as

$$
\left[\begin{array}{c}
\boldsymbol{\varepsilon}_{i} \\
\boldsymbol{\eta}_{i}
\end{array}\right] \sim \operatorname{MVN}\left(\left[\begin{array}{l}
\mathbf{0} \\
\boldsymbol{\alpha}
\end{array}\right],\left[\begin{array}{lr}
\boldsymbol{\Theta}_{i} & \mathbf{0} \\
\mathbf{0} & \boldsymbol{\Psi}
\end{array}\right]\right),
$$

where $\boldsymbol{\alpha}$ is a $q \times 1$ vector of growth factor means, $\boldsymbol{\Psi}$ is the $q \times q$ variancecovariance matrix of the growth factors. When coupled with random effects, the time-specific residuals often follow a simple structure like a mutually independent homogenous error structure (i.e., $\Theta=\sigma^{2} \mathbf{I}_{p}$ - used throughout the remainder of the study), although any number of other structures could be specified (see, e.g., 


\section{ET AL}

Jennrich \& Schluchter, 1986). Because of the normality assumption for the residuals and growth factors the probability density of $\mathbf{y}_{i}$ is also multivariate normal

$$
f\left(\mathbf{y}_{i}\right)=\phi\left[\mathbf{y}_{i} \mid \boldsymbol{\mu}(\boldsymbol{\theta}), \boldsymbol{\Sigma}(\boldsymbol{\theta})\right],
$$

where the mean vector and covariance structure follow the latent growth model such that,

$$
\boldsymbol{\mu}(\boldsymbol{\theta})=\boldsymbol{\Lambda} \boldsymbol{\alpha}_{k} \quad \text { and } \quad \boldsymbol{\Sigma}_{k}\left(\boldsymbol{\theta}_{k}\right)=\boldsymbol{\Lambda} \boldsymbol{\Psi}_{k} \Lambda^{\prime}+\boldsymbol{\Theta}_{k}
$$

and $\theta$ is the vector of parameters from all model matrices.

Muthén (2001) extended the traditional latent growth model to include finite mixtures by permitting the estimation of $K$ classes each having its own latent growth model with class-specific parameters. The density of $\mathbf{y}_{i}$ would then follow a finite mixture of normal distributions of the form

$$
f\left(\mathbf{y}_{i}\right)=\pi_{k} \phi_{k}\left[\mathbf{y}_{i} \mid \boldsymbol{\mu}_{k}\left(\boldsymbol{\theta}_{k}\right), \boldsymbol{\Sigma}_{k}\left(\boldsymbol{\theta}_{k}\right)\right]
$$

where $\pi_{k}$ is the proportion of observations arising from latent class $k$. The modelimplied mean vector and covariance matrix of a latent growth model again govern each class distribution (Bauer, 2007):

$$
\boldsymbol{\mu}_{k}\left(\boldsymbol{\theta}_{k}\right)=\Lambda \boldsymbol{\alpha}_{k} \quad \text { and } \quad \boldsymbol{\Sigma}_{k}\left(\boldsymbol{\theta}_{k}\right)=\boldsymbol{\Lambda} \boldsymbol{\Psi}_{k} \boldsymbol{\Lambda}^{\prime}+\boldsymbol{\Theta}_{k}
$$

The growth factor covariance matrices and residual covariance matrices are often presumed to be invariant over classes (i.e., $\boldsymbol{\Psi}_{k}=\boldsymbol{\Psi}$ and $\boldsymbol{\Theta}_{k}=\boldsymbol{\Theta}$ for all $k$ ). Thus, the only differences between classes are in the model-implied means of the repeated measures as determined by the class-varying growth factor means, $\boldsymbol{\alpha}_{k}$. As Hipp and Bauer (2006) pointed out, an advantage of making the within-class covariance matrices invariant is that ensures the absence of singularities, and ensures the existence of a global solution. 


\section{MPLUS IN GMM ESTIMATION}

\section{The EM Algorithm}

The EM algorithm is an iterative optimization strategy for finding ML parameter estimates by reformulating the given incomplete data or missing data problem as a complete-data problem (McLachlan \& Krishnan, 2008). The algorithm iterates between two steps - an expectation (E) step and a maximization (M) step and then iteratively repeats this sequence until some convergence criteria is met (see, e.g., Harring, 2012; Liu, 2012; Muthén \& Shedden, 1999 for a complete description of the algorithm).

\section{Simulation Design}

In this study two Monte Carlo simulations were conducted to help understand the boundaries under which GMM parameters might be successfully estimated within Mplus. The conditions manipulated under Simulation 1, which are displayed in Table 1, include: starting value quality (SVQ), number of random starts (RS), number of final optimizations (FO), perturbation level of the starting values (PL), convergence criterion for the EM algorithm (MCONV), and model complexity (MC). A second smaller simulation study was conducted where the data generation model and the estimation model were identical and only the population values for model parameters were used as starting values. Also, only a three-class model (the correct model) was fitted under Simulation 2. Therefore, the main differences between Simulations 1 and 2 are the model complexities considered and the starting values that were used. Although this second simulation design was thought to be unrealistic in practice (because the true number of mixing distributions is unknown nor are the parameter values), it provided a "best case scenario" from which to compare all other non-optimal conditions.

Table 1. Conditions and Levels of Manipulated Factors for Simulation 1

\begin{tabular}{ll} 
Conditions & Levels \\
\hline Starting value quality (SVQ) & Mplus default values, LGM ${ }^{a}$ and LCGM L $^{\text {output values }}$ \\
Number of random starts (RS) & $25,50,100,200$ \\
Number of final optimizations (FO) & $5,10,25$ \\
Perturbation level (PL) & $1,3,5,7,9$ \\
Convergence criterion (MCONV) & $1 \mathrm{E}-5,1 \mathrm{E}-8$ \\
Model complexity (MC) & 2,3, and 4-class models \\
\hline
\end{tabular}

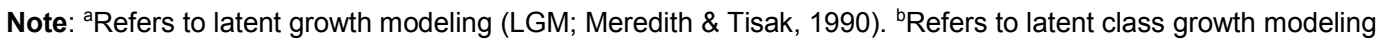
(LCGM; Nagin, 1999) 


\section{ET AL}

The population model follows a three-class linear model following conditions outlined by Tolvanen (2008) and detailed in the Appendix to this paper. Data were generated in $R$ software following the two-step procedure outlined by Hipp and Bauer (2006) and all models were fitted using Mplus 6.2 (Muthén \& Muthén, 2010). Two hundred and fifty replications were run for each of the $2 \times 4$ $\times 3 \times 5 \times 2 \times 3=720$ cells in Simulation 1 as well as each of the $4 \times 3 \times 5 \times 2=$ 120 cells in Simulation 2 in a full factorial design.

Starting value quality is defined as initial parameter estimates that were thought to be in the neighborhood of the solution found via ML. Good starting values for the means of the growth parameters are defined coming from a latent class growth analysis (Jones \& Nagin, 2007) and covariance parameters coming from fitting a one-class model, or LGM. This is aligned with what is believed as a reasonable approach to fitting GMMs in practice. Poor starting value quality is defined as the Mplus default values with no actual values given in the input file. Model complexity is measured by fitting a number of classes differing from the 3class population model. Thus for each replicate data set 2, 3, and 4-class models were fitted.

Using multiple starting values has been recommended as a method to combat convergence to a local solution prevalent when estimating GMMs. Mplus allows the number of initial stage random sets of starting values to vary and 4 levels were examined which are 25, 50, 100, and 200 (the Mplus default value is 20 with recommendations for greater number of initial random starts). It was expected that there would be an interaction between model complexity and the necessity to increase multiple starting values. Mplus also allows starting values to be perturbed randomly with the magnitude of perturbation controlled by the analyst using the STSCALE command. Five perturbation levels ranging from 1 (small perturbation) to 9 (large perturbation) with the default of 5 were examined. If local solutions are present in the analysis, changing the number of final initial solutions to analyze may impact the ability of the program to conclude that a global maximization had been reached. In terms of the convergence criteria used for the EM algorithm, pilot simulations were run using convergence criteria of 1E-5 (the Mplus default criterion), 1E-8, and 1E-10, and results showed no significant mean outcome differences between using 1E-8 and 1E-10. So, two levels of convergence criterion were used: $1 \mathrm{E}-5$ and 1E-8. Equal proportions were assumed across classes and held constant (i.e., 3-class 0.33/0.33/0.33). The sample size was fixed at $n=900$, which is in the range of past GMM simulation studies (Hamilton, 2009). 


\section{MPLUS IN GMM ESTIMATION}

\section{Outcomes}

A factorial ANOVA was used to examine the influence of the manipulated factors and their combinations on 4 outcome measures averaged over the 250 replications for the two simulations. Outcome 1 (Dlog), the proportion of number of different loglikelihood values to the total number of loglikelihood values, is expected to be low for convergence to a proper solution. Outcome 2 (Logmatch) is the percentage of replications where the highest loglikelihood solution is also the most frequently occurring solution, and is expected to be high for good model convergence. Outcome 3 is measured as the percentage of non-converged solutions defined by negative variances (Negvariance) and/or nonconvergence (Nonconverge), and Outcome 4 (Localmax) is the percentage of local maximum likelihood solutions. Both Outcomes 3 and 4 are expected to be low for convergence to a proper solution.

\section{Results}

To better understand which factors and/or combination of factors impacted model convergence and global optima, a factorial ANOVA was utilized where the four outcome variables were modeled as functions of the manipulated simulation conditions. Results for up to 5-way interactions for Simulation 1 and up to 3-way interactions for Simulation 2 were assessed and are reported separately for each of the outcomes. Only the effects of the manipulated factors were interpreted if they were identified to be both statistically significant ( $p$-value $\leq 0.05$ ) and have an effect size of $\eta^{2} \geq 0.06$ (see, e.g., Cohen, 1988, p. 283; Kohli, 2010).

Table 2 below summarizes the significant main and interaction effects for both simulation studies. Obviously, SVQ did not significantly affect the outcome variables under Simulation 1. This result was different from the findings of Jones and Nagin (2007) that using good starting values from the means of the growth parameters coming from a latent class growth analysis helps avoid the local maxima issue. Under Simulation 1, MC had the largest effect on Logmatch $\left(\hat{\eta}^{2}\right.$ $=.39)$, Negvariance $\left(\hat{\eta}^{2}=.91\right)$ and Localmax $\left(\hat{\eta}^{2}=.26\right)$. For both simulation studies, Dlog, Logmatch and Localmax were all impacted by the main effects of RS and PL. FO had significant main effects on Dlog $\left(\hat{\eta}^{2}=.30\right)$, Logmatch $\left(\hat{\eta}^{2}\right.$ $=.10)$ and Localmax $\left(\hat{\eta}^{2}=.12\right)$ under Simulation 1 but did not show significant effect on Logmatch in Simulation 2. Because population parameters were used as starting values to fit the generated model, it is not surprising that no cases of 


\section{ET AL}

nonconvergence were identified in Simulation 2. Finally, MCONV was not a significant factor for either of the two simulation studies. In terms of the interaction effects, only 2 and 3-way interaction effects were recognized for both studies. Significant 2-way interaction effect $(\mathrm{PL} \times \mathrm{MC})$ on Dlog and Localmax and 3-way interaction effect $(\mathrm{MC} \times \mathrm{PL} \times \mathrm{RS})$ on Nonconverge were found in Simulation 1. For Simulation 2, significant two-way interaction effects were found for PL $\times$ FO on Dlog, Logmatch and Localmax, for PL $\times$ RS on Logmatch and Localmax, and for RS $\times$ FO on Localmax. Significant 3-way interaction effect was obtained on Localmax for RS $\times \mathrm{FO} \times \mathrm{PL}$.

Table 2. Proportion of Variance Explained by the Outcome Variables

\begin{tabular}{|c|c|c|c|c|c|c|c|c|c|}
\hline \multirow{2}{*}{$\begin{array}{l}\text { Reported } \\
\text { Effects }\end{array}$} & \multicolumn{5}{|c|}{ Simulation 1} & \multirow{2}{*}{$\begin{array}{l}\text { Reported } \\
\text { Effects }\end{array}$} & \multicolumn{3}{|c|}{ Simulation 2} \\
\hline & Dlog & $\begin{array}{l}\text { Log- } \\
\text { match }\end{array}$ & $\begin{array}{c}\text { Neg- } \\
\text { variance }\end{array}$ & $\begin{array}{c}\text { Non- } \\
\text { convergence }\end{array}$ & Localmax & & Dlog & $\begin{array}{l}\text { Log- } \\
\text { match }\end{array}$ & $\begin{array}{l}\text { Local- } \\
\max \end{array}$ \\
\hline RS & $8.9 \%$ & $7.3 \%$ & & & $6.6 \%$ & RS & $18.9 \%$ & $8.2 \%$ & $14.1 \%$ \\
\hline FO & $29.5 \%$ & $9.9 \%$ & & & $12.3 \%$ & $\mathrm{FO}$ & $25.2 \%$ & & $6.3 \%$ \\
\hline PL & $19.0 \%$ & $11.9 \%$ & & & $10.7 \%$ & PL & $40.3 \%$ & $59.7 \%$ & $10.4 \%$ \\
\hline $\mathrm{MC}$ & $23.7 \%$ & $39.2 \%$ & $90.6 \%$ & & $26.5 \%$ & & & & \\
\hline $\mathrm{PL} \times \mathrm{MC}$ & $6.2 \%$ & & & & $9.3 \%$ & PL×FO & $6.7 \%$ & $11.4 \%$ & $10.0 \%$ \\
\hline \multirow[t]{3}{*}{$M C \times P L \times R S$} & & & & $6.4 \%$ & & $P L \times R S$ & & $8.8 \%$ & $23.1 \%$ \\
\hline & & & & & & $\mathrm{RS} \times \mathrm{FO}$ & & & $13.8 \%$ \\
\hline & & & & & & $\mathrm{FO} \times \mathrm{PL} \times \mathrm{RS}$ & & & $22.2 \%$ \\
\hline
\end{tabular}

\section{Results for the Main Effects}

Tukey's HSD procedure was used for comparing pairs of means for the main effects for both simulation studies. Means for groups in homogeneous subsets are displayed below in Tables 3 through 6 . The results presented in Table 3 show that the two simulation studies had the exact same change of directions in Dlog, Logmatch and Localmax values when the level of RS was changed. As RS increased, Dlog values decreased from .329 to .241 (for Simulation 1) and from .299 to .153 (for Simulation 2). Localmax values also decreased from .137 to .051 (for Simulation 1) and from .086 to .002 (for Simulation 2). The values of Logmatch increased from .592 to .786 for Simulation 1 and from .696 to .914 for Simulation 2. In Table 4, for both simulation studies, it was found that as FO increased, the Dlog values also increased in magnitude whereas Localmax values decreased in magnitude. Logmatch values decreased from .779 to .572 as FO 


\section{MPLUS IN GMM ESTIMATION}

increased in Simulation 2. The main effect of PL on Dlog, Logmatch, and Localmax is a little more complex. Table 5 shows that for both simulation studies, as PL increased, the Dlog values also increased. The lowest Dlog values of .194 (Simulation 1) and .110 (Simulation 2) were found at level 1 of PL. In terms of the effect of PL on Logmatch and Localmax values, Simulation 1 showed the highest value of .755 at level 2 and 3 of PL and the lowest value of .505 at level 5 of PL. Simulation 1 also had the lowest Localmax value of .052 at level 3 of PL and the highest value of .171 at level 5 of PL. For Simulation 2, increasing PL lead to decreased Logmatch values from .985 to .418 and increased Localmax values from .003 to .081. Comparing pairs of means for the main effect of MC on Dlog, Logmatch, Negvariance, and Localmax for Simulation 1 (see Table 6) suggests that as MC increased, Dlog value also increased from .209 to .370. The highest Localmax value (.177) and the lowest Logmatch value (.548) were both found at the highest level of MC. Intuitively, it seems reasonable to expect that the highest Logmatch value of .923 and the lowest Localmax value of .017 were reached for level 2 of MC (i.e., the 3-class model) because it was the model used for data generation. Results in Table 6 also indicated that Negvariance was greater for the highest MC level than for the other MC levels.

Table 3. Pairwise Comparisons among levels of RS for dependent variables: Dlog, Logmatch and Localmax

\begin{tabular}{|c|c|c|c|c|c|c|c|c|c|c|c|c|c|}
\hline \multirow{2}{*}{$\mathrm{N}$} & \multirow{2}{*}{ RS } & \multicolumn{4}{|c|}{ Subset(Dlog) } & \multicolumn{4}{|c|}{ Subset(Logmatch) } & \multicolumn{4}{|c|}{ Subset(Localmax) } \\
\hline & & 1 & 2 & 3 & 4 & 1 & 2 & 3 & 4 & 1 & 2 & 3 & 4 \\
\hline \multicolumn{14}{|c|}{ Simulation 1} \\
\hline 180 & 1 & & & & .329 & .592 & & & & & & & .137 \\
\hline 180 & 2 & & & .294 & & & .644 & & & & & .102 & \\
\hline 180 & 3 & & .256 & & & & & .713 & & & .069 & & \\
\hline 180 & 4 & .241 & & & & & & & .786 & .051 & & & \\
\hline \multicolumn{14}{|c|}{ Simulation 2} \\
\hline 30 & 1 & & & & .299 & .696 & & & & & & & .086 \\
\hline 30 & 2 & & & .249 & & & .805 & & & & & .008 & \\
\hline 30 & 3 & & .195 & . & & & & .856 & & & .004 & & \\
\hline 30 & 4 & .153 & & & & & & & .914 & .002 & & & \\
\hline
\end{tabular}




\section{ET AL}

Table 4. Pairwise Comparisons among levels of FO for dependent variables: Dlog, Logmatch and Localmax

\begin{tabular}{|c|c|c|c|c|c|c|c|c|c|c|}
\hline \multirow{2}{*}{$\mathbf{N}$} & \multirow{2}{*}{ FO } & \multicolumn{3}{|c|}{ Subset(Dlog) } & \multicolumn{3}{|c|}{ Subset(Logmatch) } & \multicolumn{3}{|c|}{ Subset(Localmax) } \\
\hline & & 1 & 2 & 3 & 1 & 2 & 3 & 1 & 2 & 3 \\
\hline \multicolumn{11}{|c|}{ Simulation 1} \\
\hline 180 & 1 & .188 & & & & & .779 & & & .146 \\
\hline 180 & 2 & & .256 & & & .700 & & & .088 & \\
\hline 180 & 3 & & & .376 & .572 & & & .035 & & \\
\hline \multicolumn{11}{|c|}{ Simulation 2} \\
\hline 40 & 1 & .155 & & & - & - & - & & & .058 \\
\hline 40 & 2 & & .208 & & - & - & - & & .015 & \\
\hline 40 & 3 & & & .309 & - & - & - & .002 & & \\
\hline
\end{tabular}

Note: No subset (Logmatch) values under Simulation 2 were provided because no significant main effect on Logmatch was found.

Table 5. Pairwise Comparisons among levels of PL for dependent variables: Dlog, Logmatch, and Localmax

\begin{tabular}{|c|c|c|c|c|c|c|c|c|c|c|c|c|c|c|c|}
\hline \multirow[b]{2}{*}{$\mathbf{N}$} & \multirow[b]{2}{*}{ PL } & \multicolumn{5}{|c|}{ Subset(Dlog) } & \multicolumn{4}{|c|}{ Subset(Logmatch) } & \multicolumn{5}{|c|}{ Subset(Localmax) } \\
\hline & & 1 & 2 & 3 & 4 & 5 & 1 & 2 & 3 & 4 & 1 & 2 & 3 & 4 & 5 \\
\hline \multicolumn{16}{|c|}{ Simulation 1} \\
\hline 144 & 1 & .194 & & & & & & & .725 & & & & & .083 & \\
\hline 144 & 2 & & .220 & & & & & & & .755 & & .065 & & & \\
\hline 144 & 3 & & & .266 & & & & & & .755 & .052 & & & & \\
\hline 144 & 4 & & & & .326 & & & .679 & & & & & .079 & & \\
\hline 144 & 5 & & & & & .360 & .505 & & & & & & & & .171 \\
\hline \multicolumn{16}{|c|}{ Simulation 2} \\
\hline 24 & 1 & .110 & & & & & & & & .985 & .003 & & & & - \\
\hline 24 & 2 & & .156 & & & & & & & .982 & .002 & & & & - \\
\hline 24 & 3 & & & .237 & & & & & .950 & & & .006 & & & - \\
\hline 24 & 4 & & & & .295 & & & .753 & & & & & .031 & & - \\
\hline 24 & 5 & & & & & .322 & .418 & & & & & & & .081 & - \\
\hline
\end{tabular}

Note: No fifth subset of Localmax was provided because only 4 subsets were identified. 


\section{MPLUS IN GMM ESTIMATION}

Table 6. Pairwise Comparisons among levels of $\mathrm{MC}$ for dependent variables: Dlog, Logmatch, Negvariance, and Localmax for Simulation 1

\begin{tabular}{ccccccccccccc} 
& & \multicolumn{2}{c}{$\begin{array}{c}\text { Subset } \\
\text { (Dlog) }\end{array}$} & & \multicolumn{2}{c}{$\begin{array}{c}\text { Subset } \\
\text { (Logmatch) }\end{array}$} & \multicolumn{3}{c}{$\begin{array}{c}\text { Subset } \\
\text { (Negvariance) }\end{array}$} & \multicolumn{3}{c}{$\begin{array}{c}\text { Subset } \\
\text { (Localmax) }\end{array}$} \\
$\mathbf{N}$ & MC & $\mathbf{1}$ & $\mathbf{2}$ & $\mathbf{3}$ & $\mathbf{1}$ & $\mathbf{2}$ & $\mathbf{3}$ & $\mathbf{1}$ & $\mathbf{2}$ & $\mathbf{1}$ & $\mathbf{2}$ & $\mathbf{3}$ \\
\hline 180 & 1 & .209 & & & & .581 & & .000 & & & .074 & \\
180 & 2 & & .240 & & & & .923 & .000 & & .017 & & \\
180 & 3 & & & .370 & .548 & & & & .029 & & & .177 \\
\hline
\end{tabular}

\section{Results for the Interaction Effects}

Simple main effect pairwise comparisons were conducted to investigate the nature of significant two and three-way interaction effects for each of the simulation studies. For those significant interaction effects with a clear pattern, graphics were provided.

The only significant two-way interaction effect found in Simulation 1 was PL $\times$ MC for dependent variables: Dlog and Localmax. Three significant two-way interaction effects in Simulation 2 were PL $\times$ FO (for dependent variables: Dlog, Logmatch and Localmax), PL $\times$ RS (for dependent variables: Logmatch and Localmax), and RS $\times$ FO (for dependent variable: Localmax). Tables 7 and 8 present simple main effect results related to the PL $\times \mathrm{MC}$ interaction on Dlog and Localmax respectively under Simulation 1.

For Dlog, no significant mean differences were found between levels of MC at level 1 of PL. Significant mean differences were found between level 3 and level 1 and between level 3 and level 2 of MC at level 2, 3 and 4 of PL. Significant mean differences were also found for all pairs of MC levels at level 5 of PL. Further, level 3 of MC always had the highest Dlog values $(.238, .296, .357, .460$, and .501$)$ across all PL levels compared with the other two MC levels, and Dlog values showed the slowest increase for level 1 of MC starting from level 3 of PL $(0.207,0.217$, and 0.233). Clearly, MC is an important factor affecting Dlog measures at all PL levels.

Another important observation is that as PL increased in magnitude, Dlog increased for level 2 and 3 of MC. Dlog started to increase for level 1 of MC from level 3 of PL. Therefore, a high PL level might not be a good choice for a low Dlog solution for any of the models that were considered. In terms of the PL $\times$ $\mathrm{MC}$ interaction effect on Localmax, it may be observed from the results reported 


\section{ET AL}

in Table 8 that the lowest Localmax mean values $(.008, .004, .005$, and .020$)$ were always associated with level 2 of MC for each of the levels of PL, which seems reasonable because level 2 of $\mathrm{MC}$ is the 3-class model used for data generation. The lowest Localmax values were found with level 1 and 2 of MC at PL level 3 and level 3 of MC at PL level 2. It may also be noted that Localmax values started to increase markedly in magnitude at and above PL level 4 for each of the levels of MC, particularly with level 3 of MC (.186 at PL level 4 and .349 at PL level 5).

Table 7. Simple Main Effect Pairwise Comparisons Corresponding to PL $\times$ MC Interaction for Dlog (Simulation 1)

\begin{tabular}{|c|c|c|c|c|c|c|}
\hline \multirow{2}{*}{\multicolumn{2}{|c|}{$\begin{array}{l}\text { Mean } 1 \\
\text { Factor Level }\end{array}$}} & \multicolumn{4}{|c|}{ Mean 2} & \multirow{3}{*}{$\begin{array}{c}\text { Mean Difference } \\
\text { (Mean } 1 \text { - Mean 2) }\end{array}$} \\
\hline & & & \multicolumn{3}{|c|}{ Factor Level } & \\
\hline $\mathrm{PL}$ & MC & $\bar{X}_{P L=j, M C=k}$ & PL & MC & $\bar{X}_{P L=j^{\prime}, M C=k^{\prime}}$ & \\
\hline 1 & 1 & 0.196 & 1 & 2 & 0.149 & 0.047 \\
\hline 1 & 1 & 0.196 & 1 & 3 & 0.238 & -0.042 \\
\hline 1 & 2 & 0.149 & 1 & 3 & 0.238 & -0.089 \\
\hline 2 & 1 & 0.193 & 2 & 2 & 0.170 & 0.023 \\
\hline 2 & 1 & 0.193 & 2 & 3 & 0.296 & $-0.103^{*}$ \\
\hline 2 & 2 & 0.170 & 2 & 3 & 0.296 & $-0.126^{*}$ \\
\hline 3 & 1 & 0.207 & 3 & 2 & 0.234 & -0.027 \\
\hline 3 & 1 & 0.207 & 3 & 3 & 0.357 & $-0.150^{*}$ \\
\hline 3 & 2 & 0.234 & 3 & 3 & 0.357 & $-0.123^{*}$ \\
\hline 4 & 1 & 0.217 & 4 & 2 & 0.303 & -0.086 \\
\hline 4 & 1 & 0.217 & 4 & 3 & 0.460 & $-0.243^{*}$ \\
\hline 4 & 2 & 0.303 & 4 & 3 & 0.460 & $-0.157^{*}$ \\
\hline 5 & 1 & 0.233 & 5 & 2 & 0.346 & $-0.113^{*}$ \\
\hline 5 & 1 & 0.233 & 5 & 3 & 0.501 & $-0.268^{*}$ \\
\hline 5 & 2 & 0.346 & 5 & 3 & 0.501 & $-0.155^{\star}$ \\
\hline
\end{tabular}

Note: The increased Dlog values for level 1 of MC at level 3, 4, and 5 level of PL and the increased Dlog values for level 3 of MC across levels of PL are in boldface.

*significant at $\alpha=.05$ level. Family-Wise Error (FWE) was separately controlled at each level of perturbation at which simple main effect tests were performed. 


\section{MPLUS IN GMM ESTIMATION}

Table 8. Simple Main Effect Pairwise Comparisons Corresponding to $\mathrm{PL} \times \mathrm{MC}$ Interaction for Localmax (Simulation 1)

\begin{tabular}{|c|c|c|c|c|c|c|}
\hline \multirow{2}{*}{\multicolumn{2}{|c|}{$\begin{array}{l}\text { Mean } 1 \\
\text { Factor Level }\end{array}$}} & \multicolumn{4}{|c|}{ Mean 2} & \multirow{3}{*}{$\begin{array}{c}\text { Mean Difference } \\
\text { (Mean } 1 \text { - Mean 2) }\end{array}$} \\
\hline & & \multirow[b]{2}{*}{$\bar{X}_{P L=j, M C=k}$} & \multicolumn{2}{|c|}{ Factor Level } & \multirow[b]{2}{*}{$\bar{X}_{P L=j^{\prime}, M C=k^{\prime}}$} & \\
\hline $\mathrm{PL}$ & $\mathrm{MC}$ & & PL & $\mathrm{MC}$ & & \\
\hline 1 & 1 & 0.118 & 1 & 2 & 0.008 & $0.110^{*}$ \\
\hline 1 & 1 & 0.118 & 1 & 3 & 0.123 & -0.005 \\
\hline 1 & 2 & 0.008 & 1 & 3 & 0.123 & $-0.115^{*}$ \\
\hline 2 & 1 & 0.083 & 2 & 2 & 0.004 & 0.079 \\
\hline 2 & 1 & 0.083 & 2 & 3 & 0.108 & -0.025 \\
\hline 2 & 2 & 0.004 & 2 & 3 & 0.108 & $-0.104^{*}$ \\
\hline 3 & 1 & 0.028 & 3 & 2 & 0.005 & 0.023 \\
\hline 3 & 1 & 0.028 & 3 & 3 & 0.122 & $-0.094^{*}$ \\
\hline 3 & 2 & 0.005 & 3 & 3 & 0.122 & $-0.117^{*}$ \\
\hline 4 & 1 & 0.030 & 4 & 2 & 0.020 & 0.010 \\
\hline 4 & 1 & 0.030 & 4 & 3 & 0.186 & $-0.156^{*}$ \\
\hline 4 & 2 & 0.020 & 4 & 3 & 0.186 & $-0.166^{*}$ \\
\hline 5 & 1 & 0.112 & 5 & 2 & 0.052 & 0.060 \\
\hline 5 & 1 & 0.112 & 5 & 3 & 0.349 & $-0.237^{*}$ \\
\hline 5 & 2 & 0.052 & 5 & 3 & 0.349 & $-0.297^{*}$ \\
\hline
\end{tabular}

Note: The increased Localmax values for 3 levels of MC at level 4 and level 5 of PL are in boldface.

*significant at $\alpha=.05$ level. Family-Wise Error (FWE) was separately controlled at each level of perturbation at which simple main effect tests were performed.

Graphic presentations of the interaction effect of PL $\times$ FO for dependent variables Dlog, Logmatch and Localmax (from Simulation 2) are provided in Figures 1 through 3. It may be observed that as PL increased, Dlog and Localmax both increased whereas Logmatch decreased for all FO levels. The highest Dlog, the lowest Logmatch and the lowest Localmax values were seen with level 3 of FO at higher PL levels. It also may be observed in Figures 2 and 3 that Logmatch and Localmax values were generally stable and similar in magnitude for pairs of FO levels at PL levels 1 and 3. However, starting from level 4 of PL, Logmatch and Localmax values both showed a sudden change, with Logmatch values dropping and Localmax rising sharply. The results reported in Tables 9 through 


\section{ET AL}

11 show the nature of the interaction between PL and FO on Dlog, Logmatch and Localmax. Significant Dlog mean differences were found between level 1 and level 3 of FO across PL levels 2-5 and there were no significant mean differences between level 1 and level 2 of FO at any PL levels (see Table 9). Tables 10 and 11 both show most significant mean differences in Logmatch and Localmax occurred at level 5 of PL between pairs of FO levels.

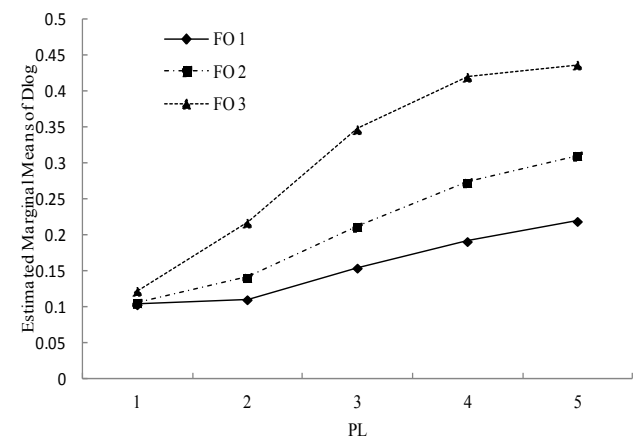

Figure 1. PL $\times$ FO For Outcome 1 (Dlog)

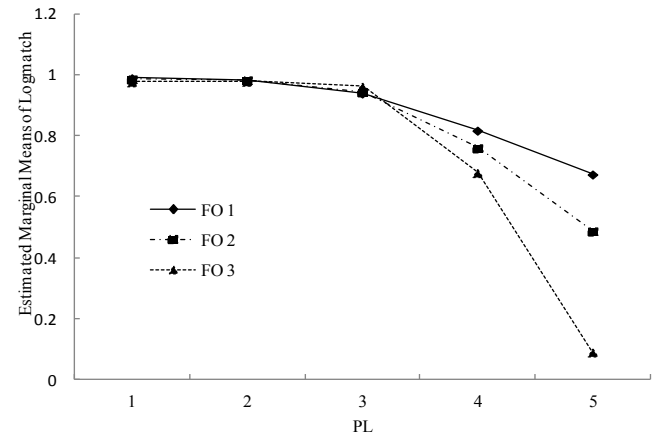

Figure 2. PL $\times$ FO Outcome 2 (Logmatch)

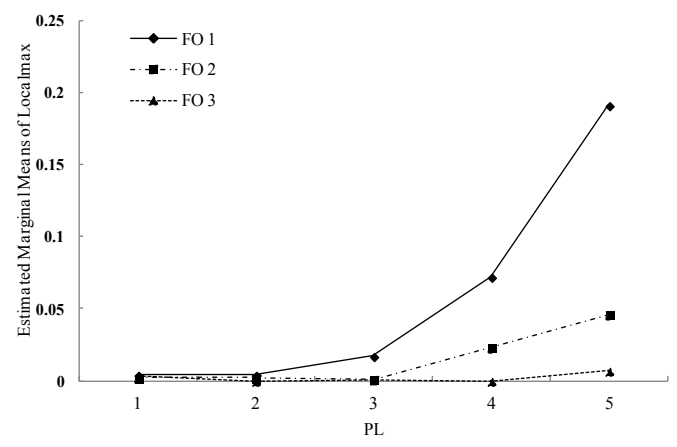

Figure 3. PL $\times$ FO Outcome 4 (Localmax) 


\section{MPLUS IN GMM ESTIMATION}

Table 9. Simple Main Effect Pairwise Comparisons Corresponding to PL $\times$ FO Interaction for Dlog (Simulation 2)

\begin{tabular}{|c|c|c|c|c|c|c|}
\hline \multirow{2}{*}{\multicolumn{2}{|c|}{$\begin{array}{l}\text { Mean } 1 \\
\text { Factor Level }\end{array}$}} & \multicolumn{4}{|c|}{ Mean 2} & \multirow{3}{*}{$\begin{array}{c}\text { Mean Difference } \\
\text { (Mean } 1 \text { - Mean 2) }\end{array}$} \\
\hline & & & \multicolumn{3}{|c|}{ Factor Level } & \\
\hline PL & FO & $\bar{X}_{P L=j, M C=k}$ & $\mathrm{PL}$ & $\mathrm{FO}$ & $\bar{X}_{P L=j^{\prime}, M C=k^{\prime}}$ & \\
\hline 1 & 1 & 0.103 & 1 & 2 & 0.105 & -0.002 \\
\hline 1 & 1 & 0.103 & 1 & 3 & 0.122 & -0.019 \\
\hline 1 & 2 & 0.105 & 1 & 3 & 0.122 & -0.017 \\
\hline 2 & 1 & 0.110 & 2 & 2 & 0.141 & -0.031 \\
\hline 2 & 1 & 0.110 & 2 & 3 & 0.217 & $-0.107^{\star}$ \\
\hline 2 & 2 & 0.141 & 2 & 3 & 0.217 & -0.076 \\
\hline 3 & 1 & 0.154 & 3 & 2 & 0.211 & -0.057 \\
\hline 3 & 1 & 0.154 & 3 & 3 & 0.347 & $-0.193^{*}$ \\
\hline 3 & 2 & 0.211 & 3 & 3 & 0.347 & $-0.136^{*}$ \\
\hline 4 & 1 & 0.191 & 4 & 2 & 0.273 & -0.082 \\
\hline 4 & 1 & 0.191 & 4 & 3 & 0.420 & $-0.229^{*}$ \\
\hline 4 & 2 & 0.273 & 4 & 3 & 0.420 & $-0.147^{*}$ \\
\hline 5 & 1 & 0.219 & 5 & 2 & 0.310 & -0.091 \\
\hline 5 & 1 & 0.219 & 5 & 3 & 0.437 & $-0.218^{*}$ \\
\hline 5 & 2 & 0.310 & 5 & 3 & 0.437 & $-0.127^{*}$ \\
\hline
\end{tabular}

Note: *significant at $\alpha=.05$ level. Family-Wise Error (FWE) was separately controlled at each level of perturbation at which simple main effect tests were performed. 


\section{ET AL}

Table 10. Simple Main Effect Pairwise Comparisons Corresponding to PL $\times$ FO Interaction for Logmatch (Simulation 2)

\begin{tabular}{|c|c|c|c|c|c|c|}
\hline \multirow{2}{*}{\multicolumn{2}{|c|}{$\begin{array}{l}\text { Mean } 1 \\
\text { Factor Level }\end{array}$}} & \multicolumn{4}{|c|}{ Mean 2} & \multirow{3}{*}{$\begin{array}{c}\text { Mean Difference } \\
\text { (Mean } 1 \text { - Mean 2) }\end{array}$} \\
\hline & & & \multicolumn{3}{|c|}{ Factor Level } & \\
\hline & FO & $\bar{X}_{P L=j, M C=k}$ & $\mathrm{PL}$ & FO & $\bar{X}_{P L=j^{\prime}, M C=k^{\prime}}$ & \\
\hline 1 & 1 & 0.991 & 1 & 2 & 0.987 & 0.004 \\
\hline 1 & 1 & 0.991 & 1 & 3 & 0.978 & 0.013 \\
\hline 1 & 2 & 0.987 & 1 & 3 & 0.978 & 0.009 \\
\hline 2 & 1 & 0.981 & 2 & 2 & 0.984 & -0.003 \\
\hline 2 & 1 & 0.981 & 2 & 3 & 0.980 & 0.001 \\
\hline 2 & 2 & 0.984 & 2 & 3 & 0.980 & 0.004 \\
\hline 3 & 1 & 0.941 & 3 & 2 & 0.945 & -0.004 \\
\hline 3 & 1 & 0.941 & 3 & 3 & 0.964 & -0.023 \\
\hline 3 & 2 & 0.945 & 3 & 3 & 0.964 & -0.019 \\
\hline 4 & 1 & 0.819 & 4 & 2 & 0.761 & 0.058 \\
\hline 4 & 1 & 0.819 & 4 & 3 & 0.680 & $0.139^{*}$ \\
\hline 4 & 2 & 0.761 & 4 & 3 & 0.680 & 0.081 \\
\hline 5 & 1 & 0.675 & 5 & 2 & 0.488 & $0.187^{*}$ \\
\hline 5 & 1 & 0.675 & 5 & 3 & 0.091 & $0.584^{*}$ \\
\hline 5 & 2 & 0.488 & 5 & 3 & 0.091 & $0.397^{*}$ \\
\hline
\end{tabular}

Note: *significant at $\alpha=.05$ level. Family-Wise Error (FWE) was separately controlled at each level of perturbation at which simple main effect tests were performed. 


\section{MPLUS IN GMM ESTIMATION}

Table 11. Simple Main Effect Pairwise Comparisons Corresponding to $\mathrm{PL} \times \mathrm{FO}$ Interaction for Localmax (Simuation 2)

\begin{tabular}{|c|c|c|c|c|c|c|}
\hline \multirow{2}{*}{\multicolumn{2}{|c|}{$\begin{array}{l}\text { Mean } 1 \\
\text { Factor Level }\end{array}$}} & \multicolumn{4}{|c|}{ Mean 2} & \multirow{3}{*}{$\begin{array}{c}\text { Mean Difference } \\
\text { (Mean } 1 \text { - Mean 2) }\end{array}$} \\
\hline & & & \multicolumn{3}{|c|}{ Factor Level } & \\
\hline$P L$ & $\mathrm{FO}$ & $\bar{X}_{P L=j, M C=k}$ & $\mathrm{PL}$ & FO & $\bar{X}_{P L=j^{\prime}, M C=k^{\prime}}$ & \\
\hline 1 & 1 & 0.004 & 1 & 2 & 0.002 & 0.002 \\
\hline 1 & 1 & 0.004 & 1 & 3 & 0.003 & 0.001 \\
\hline 1 & 2 & 0.002 & 1 & 3 & 0.003 & -0.001 \\
\hline 2 & 1 & 0.004 & 2 & 2 & 0.002 & 0.002 \\
\hline 2 & 1 & 0.004 & 2 & 3 & 0.000 & 0.004 \\
\hline 2 & 2 & 0.002 & 2 & 3 & 0.000 & 0.002 \\
\hline 3 & 1 & 0.017 & 3 & 2 & 0.001 & 0.016 \\
\hline 3 & 1 & 0.017 & 3 & 3 & 0.001 & 0.016 \\
\hline 3 & 2 & 0.001 & 3 & 3 & 0.001 & 0.000 \\
\hline 4 & 1 & 0.072 & 4 & 2 & 0.023 & 0.049 \\
\hline 4 & 1 & 0.072 & 4 & 3 & 0.000 & 0.072 \\
\hline 4 & 2 & 0.023 & 4 & 3 & 0.000 & 0.023 \\
\hline 5 & 1 & 0.191 & 5 & 2 & 0.046 & $0.145^{*}$ \\
\hline 5 & 1 & 0.191 & 5 & 3 & 0.007 & $0.184^{*}$ \\
\hline 5 & 2 & 0.046 & 5 & 3 & 0.007 & 0.039 \\
\hline
\end{tabular}

Note: *significant at $\alpha=.05$ level. Family-Wise Error (FWE) was separately controlled at each level of perturbation at which simple main effect tests were performed.

Figures 4 and 5 graphically depict the interaction effect between PL and RS for the dependent variables: Logmatch and Localmax in Simulation 2. At level 1 and level 2 of PL, both Logmatch and Localmax values were very similar across all RS levels. From level 3 of PL, discrepancies in Logmatch and Localmax values among the RS levels started to show up and grow even larger at level 4 and level 5 of PL. It may also be noticed that Logmatch values decreased markedly at PL level 4 for all RS levels, with the sharpest decline observed at level 1 of RS. In contrast, Localmax values increased dramatically at PL level 4 for level 1 of RS. Tables 12 and 13 were provided to confirm what had been observed. For Logmatch, most significant mean differences were found between pairs of RS levels at level 4 and level 5 of PL (see Table 12). Significant Localmax mean 


\section{ET AL}

differences were found at levels 4 and 5 of PL between level 1 and level 2, level 1 and level 3, and level 1 and level 4 of RS (see Table 13), with no significant mean differences found for pairs of levels 2,3 , and 4 of RS.

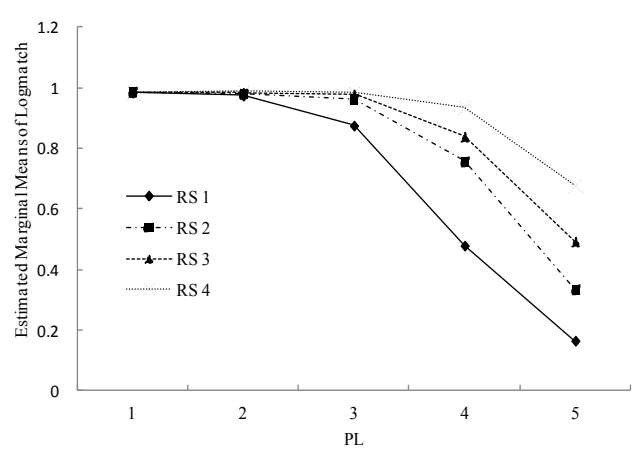

Figure 4. PL $\times$ RS for Outcome 2 (Logmatch)

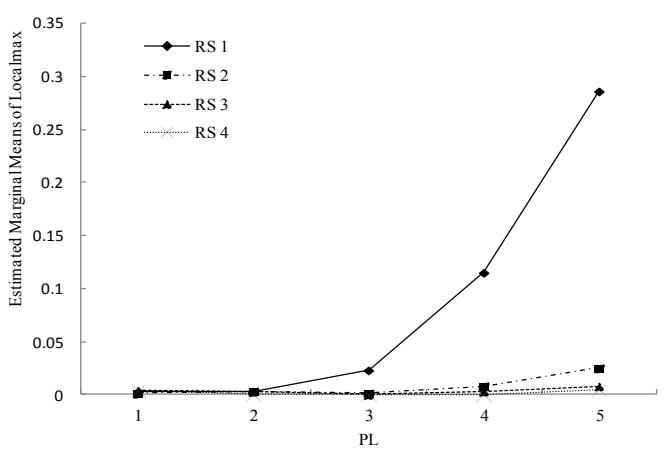

Figure 5. PL $\times$ RS for Outcome 4 (Localmax) 


\section{MPLUS IN GMM ESTIMATION}

Table 12. Simple Main Effect Pairwise Comparisons Corresponding to $P L \times R S$ Interaction for Logmatch (Simulation 2)

\begin{tabular}{|c|c|c|c|c|c|c|}
\hline \multirow{2}{*}{\multicolumn{2}{|c|}{$\begin{array}{l}\text { Mean } 1 \\
\text { Factor Level }\end{array}$}} & \multicolumn{4}{|c|}{ Mean 2} & \multirow{3}{*}{$\begin{array}{c}\text { Mean Difference } \\
\text { (Mean } 1 \text { - Mean 2) }\end{array}$} \\
\hline & & & \multicolumn{3}{|c|}{ Factor Level } & \\
\hline $\mathrm{PL}$ & $\mathrm{RS}$ & $\bar{X}_{P L=j, R S=k}$ & PL & RS & $\bar{X}_{P L=j^{\prime}, R S=k^{\prime}}$ & \\
\hline 1 & 1 & 0.984 & 1 & 2 & 0.987 & -0.003 \\
\hline 1 & 1 & 0.984 & 1 & 3 & 0.987 & -0.003 \\
\hline 1 & 1 & 0.984 & 1 & 4 & 0.984 & 0.000 \\
\hline 1 & 2 & 0.987 & 1 & 3 & 0.987 & 0.000 \\
\hline 1 & 2 & 0.987 & 1 & 4 & 0.984 & 0.003 \\
\hline 1 & 3 & 0.987 & 1 & 4 & 0.984 & 0.003 \\
\hline 2 & 1 & 0.974 & 2 & 2 & 0.980 & -0.006 \\
\hline 2 & 1 & 0.974 & 2 & 3 & 0.984 & -0.010 \\
\hline 2 & 1 & 0.974 & 2 & 4 & 0.988 & -0.014 \\
\hline 2 & 2 & 0.980 & 2 & 3 & 0.984 & -0.004 \\
\hline 2 & 2 & 0.980 & 2 & 4 & 0.988 & -0.008 \\
\hline 2 & 3 & 0.984 & 2 & 4 & 0.988 & -0.004 \\
\hline 3 & 1 & 0.875 & 3 & 2 & 0.963 & -0.088 \\
\hline 3 & 1 & 0.875 & 3 & 3 & 0.979 & $-0.104^{*}$ \\
\hline 3 & 1 & 0.875 & 3 & 4 & 0.984 & $-0.109^{*}$ \\
\hline 3 & 2 & 0.963 & 3 & 3 & 0.979 & -0.016 \\
\hline 3 & 2 & 0.963 & 3 & 4 & 0.984 & -0.021 \\
\hline 3 & 3 & 0.979 & 3 & 4 & 0.984 & -0.005 \\
\hline 4 & 1 & 0.479 & 4 & 2 & 0.757 & $-0.278^{*}$ \\
\hline 4 & 1 & 0.479 & 4 & 3 & 0.839 & $-0.360^{*}$ \\
\hline 4 & 1 & 0.479 & 4 & 4 & 0.937 & $-0.458^{*}$ \\
\hline 4 & 2 & 0.757 & 4 & 3 & 0.839 & -0.082 \\
\hline 4 & 2 & 0.757 & 4 & 4 & 0.937 & $-0.180^{*}$ \\
\hline 4 & 3 & 0.839 & 4 & 4 & 0.937 & -0.098 \\
\hline 5 & 1 & 0.165 & 5 & 2 & 0.335 & $-0.170^{*}$ \\
\hline 5 & 1 & 0.165 & 5 & 3 & 0.493 & $-0.328^{*}$ \\
\hline 5 & 1 & 0.165 & 5 & 4 & 0.679 & $-0.514^{*}$ \\
\hline 5 & 2 & 0.335 & 5 & 3 & 0.493 & $-0.158^{*}$ \\
\hline 5 & 2 & 0.335 & 5 & 4 & 0.679 & $-0.344^{*}$ \\
\hline
\end{tabular}

Note: The decreased Logmatch values for level 1 of RS at PL levels 3, 4 and 5 are in boldface.

*significant at $\alpha=.05$ level. Family-Wise Error (FWE) was separately controlled at each level of perturbation at which simple main effect tests were performed. 


\section{ET AL}

Table 13. Simple Main Effect Pairwise Comparisons Corresponding to $\mathrm{PL} \times \mathrm{RS}$ Interaction for Localmax (Simulation 2)

\begin{tabular}{|c|c|c|c|c|c|c|}
\hline \multirow{2}{*}{\multicolumn{2}{|c|}{$\begin{array}{l}\text { Mean } 1 \\
\text { Factor Level }\end{array}$}} & \multicolumn{4}{|c|}{ Mean 2} & \multirow{3}{*}{$\begin{array}{c}\text { Mean Difference } \\
\text { (Mean } 1 \text { - Mean 2) }\end{array}$} \\
\hline & & & \multicolumn{3}{|c|}{ Factor Level } & \\
\hline $\mathrm{PL}$ & $\mathrm{RS}$ & $\bar{X}_{P L=j, R S=k}$ & PL & RS & $\bar{X}_{P L=j^{\prime}, R S=k^{\prime}}$ & \\
\hline 1 & 1 & 0.003 & 1 & 2 & 0.001 & 0.002 \\
\hline 1 & 1 & 0.003 & 1 & 3 & 0.004 & -0.001 \\
\hline 1 & 1 & 0.003 & 1 & 4 & 0.004 & -0.001 \\
\hline 1 & 2 & 0.001 & 1 & 3 & 0.004 & -0.003 \\
\hline 1 & 2 & 0.001 & 1 & 4 & 0.004 & -0.003 \\
\hline 1 & 3 & 0.004 & 1 & 4 & 0.004 & 0.000 \\
\hline 2 & 1 & 0.003 & 2 & 2 & 0.003 & 0.000 \\
\hline 2 & 1 & 0.003 & 2 & 3 & 0.003 & 0.000 \\
\hline 2 & 1 & 0.003 & 2 & 4 & 0.000 & 0.003 \\
\hline 2 & 2 & 0.003 & 2 & 3 & 0.003 & 0.000 \\
\hline 2 & 2 & 0.003 & 2 & 4 & 0.000 & 0.003 \\
\hline 2 & 3 & 0.003 & 2 & 4 & 0.000 & 0.003 \\
\hline 3 & 1 & 0.023 & 3 & 2 & 0.001 & 0.022 \\
\hline 3 & 1 & 0.023 & 3 & 3 & 0.000 & 0.023 \\
\hline 3 & 1 & 0.023 & 3 & 4 & 0.000 & 0.023 \\
\hline 3 & 2 & 0.001 & 3 & 3 & 0.001 & 0.000 \\
\hline 3 & 2 & 0.001 & 3 & 4 & 0.000 & 0.001 \\
\hline 3 & 3 & 0 & 3 & 4 & 0.000 & 0.000 \\
\hline 4 & 1 & 0.115 & 4 & 2 & 0.008 & $0.107^{*}$ \\
\hline 4 & 1 & 0.115 & 4 & 3 & 0.003 & $0.112^{*}$ \\
\hline 4 & 1 & 0.115 & 4 & 4 & 0.000 & $0.115^{*}$ \\
\hline 4 & 2 & 0.008 & 4 & 3 & 0.003 & 0.005 \\
\hline 4 & 2 & 0.008 & 4 & 4 & 0.000 & 0.008 \\
\hline 4 & 3 & 0.003 & 4 & 4 & 0.000 & 0.003 \\
\hline 5 & 1 & 0.286 & 5 & 2 & 0.025 & $0.261^{*}$ \\
\hline 5 & 1 & 0.286 & 5 & 3 & 0.009 & $0.277^{*}$ \\
\hline 5 & 1 & 0.286 & 5 & 4 & 0.005 & $0.281^{*}$ \\
\hline 5 & 2 & 0.025 & 5 & 3 & 0.008 & 0.017 \\
\hline 5 & 2 & 0.025 & 5 & 4 & 0.005 & 0.020 \\
\hline
\end{tabular}

Note: The increased Localmax values for level 1 of RS at PL level 3, level 4 and level 5 are in boldface.

*significant at $\alpha=.05$ level. Family-Wise Error (FWE) was separately controlled at each level of perturbation at which simple main effect tests were performed. 


\section{MPLUS IN GMM ESTIMATION}

Figure 6 shows how RS and FO interacted for the Dependent Variable: Localmax. Obviously, the Localmax mean values were very close in magnitude among levels of FO at level 2, 3 and 4 of RS, and as RS increased, the Localmax mean values became progressively closer in magnitude for all of the levels of FO. Localmax mean differences were clear only at level 1 of RS. Table 14 followed shows significant mean differences between level 1 and level 2 and between level 1 and level 3 of FO at RS level 1. It should also be noted that from RS level 1 to RS level 2, Localmax mean values decreased in magnitude for all FO levels (with the sharpest decrease observed for level 1 of FO), suggesting a high RS is always preferred for a low Localmax for any level of FO. It also suggests that when RS is very low at level 1, a low FO level should be considered for low percentage of Localmax solutions.

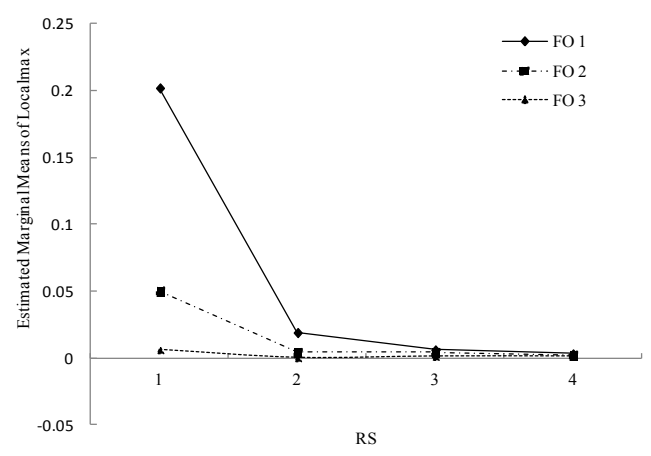

Figure 6. RS × FO for Outcome 4 (Localmax) 


\section{ET AL}

Table 14. Simple Main Effect Pairwise Comparisons Corresponding to RS $\times$ FO Interaction for Localmax (Simuation 2)

\begin{tabular}{ccccccc}
$\begin{array}{c}\text { Mean 1 } \\
\text { Factor Level } \\
\text { RS }\end{array}$ & FO & $\bar{X}_{P L=j, F O=k}$ & RS & FO & $\bar{X}_{P L=j^{\prime}, F O=k^{\prime}}$ & Mean 2 \\
\hline 1 & 1 & 0.202 & 1 & 2 & 0.050 & $0.152^{*}$ \\
1 & 1 & 0.202 & 1 & 3 & 0.006 & $0.196^{*}$ \\
1 & 2 & 0.050 & 1 & 3 & 0.006 & 0.044 \\
& & & & & & 0.015 \\
2 & 1 & 0.019 & 2 & 2 & 0.004 & 0.019 \\
2 & 1 & 0.019 & 2 & 3 & 0.000 & 0.004 \\
2 & 2 & 0.004 & 2 & 3 & 0.000 & 0.002 \\
& & & & & & 0.005 \\
3 & 1 & 0.006 & 3 & 2 & 0.004 & 0.003 \\
3 & 1 & 0.006 & 3 & 3 & 0.001 & 0.001 \\
3 & 2 & 0.004 & 3 & 3 & 0.001 & 0.002 \\
4 & 1 & 0.003 & 4 & 2 & 0.002 & 0.001 \\
\hline 4 & 1 & 0.003 & 4 & 3 & 0.001 & \\
\hline
\end{tabular}

Note: *significant at $\alpha=.05$ level. Family-Wise Error (FWE) was separately controlled at each level of number of random starts at which the simple main effect tests were performed.

Two three-way interaction effects were found to be significant at $\alpha=0.05$. One of these significant outcomes was found for the dependent variable: Nonconverge (Simulation 1) and the other for the dependent variable: Localmax (Simulation 2). Simple main effect pairwise comparisons corresponding to the two-way interaction effects from PL $\times$ RS on Nonconverge were conducted at each of the levels of MC for Simulation 1. No significant mean differences were found for the two-way interaction under either level 1 or level 2 of MC. Therefore, results for the interaction effect of PL $\times$ RS on Nonconverge under level 1 and 2 of $\mathrm{MC}$ are not reported. Table 15 shows only the interaction effect of PL $\times$ RS on Nonconverge under level 3 of MC. It can be seen that all significant mean outcome differences were found at level 5 of PL although there was no significant mean difference between level 3 and level 4 of RS. This findings would seems to suggest that with a complicated model (e.g., the 4-class model), if a high PL level is used, higher levels of RS (level 3 or level 4) should be considered to increase the number of converged solutions. 


\section{MPLUS IN GMM ESTIMATION}

Table 15. Simple Main Effect Pairwise Comparisons Corresponding to $\mathrm{PL} \times \mathrm{RS}$ Interaction for Nonconverge under Level 3 of MC

\begin{tabular}{|c|c|c|c|c|c|c|}
\hline \multirow{2}{*}{\multicolumn{2}{|c|}{$\begin{array}{l}\text { Mean } 1 \\
\text { Factor Level }\end{array}$}} & \multicolumn{4}{|c|}{ Mean 2} & \multirow{3}{*}{$\begin{array}{c}\text { Mean Difference } \\
\text { (Mean } 1 \text { - Mean 2) }\end{array}$} \\
\hline & & & \multicolumn{3}{|c|}{ Factor Level } & \\
\hline & $\mathrm{RS}$ & $\bar{X}_{P L=j, R S=k}$ & PL & RS & $\bar{X}_{P L=j^{\prime}, R S=k^{\prime}}$ & \\
\hline 1 & 1 & 0.000 & 1 & 2 & 0.000 & 0.000 \\
\hline 1 & 1 & 0.000 & 1 & 3 & 0.000 & 0.000 \\
\hline 1 & 1 & 0.000 & 1 & 4 & 0.001 & 0.000 \\
\hline 1 & 2 & 0.000 & 1 & 3 & 0.000 & 0.000 \\
\hline 1 & 2 & 0.000 & 1 & 4 & 0.001 & 0.000 \\
\hline 1 & 3 & 0.000 & 1 & 4 & 0.001 & 0.000 \\
\hline 2 & 1 & 0.000 & 2 & 2 & 0.001 & 0.000 \\
\hline 2 & 1 & 0.000 & 2 & 3 & 0.001 & 0.000 \\
\hline 2 & 1 & 0.000 & 2 & 4 & 0.000 & 0.000 \\
\hline 2 & 2 & 0.001 & 2 & 3 & 0.001 & 0.000 \\
\hline 2 & 2 & 0.001 & 2 & 4 & 0.000 & 0.001 \\
\hline 2 & 3 & 0.001 & 2 & 4 & 0.000 & 0.001 \\
\hline 3 & 1 & 0.000 & 3 & 2 & 0.000 & 0.000 \\
\hline 3 & 1 & 0.000 & 3 & 3 & 0.000 & 0.000 \\
\hline 3 & 1 & 0.000 & 3 & 4 & 0.000 & 0.000 \\
\hline 3 & 2 & 0.000 & 3 & 3 & 0.000 & 0.000 \\
\hline 3 & 2 & 0.000 & 3 & 4 & 0.000 & 0.000 \\
\hline 3 & 3 & 0.000 & 3 & 4 & 0.000 & 0.000 \\
\hline 4 & 1 & 0.000 & 4 & 2 & 0.000 & 0.000 \\
\hline 4 & 1 & 0.000 & 4 & 3 & 0.000 & 0.000 \\
\hline 4 & 1 & 0.000 & 4 & 4 & 0.000 & 0.000 \\
\hline 4 & 2 & 0.000 & 4 & 3 & 0.000 & 0.000 \\
\hline 4 & 2 & 0.000 & 4 & 4 & 0.000 & 0.000 \\
\hline 4 & 3 & 0.000 & 4 & 4 & 0.000 & 0.000 \\
\hline 5 & 1 & 0.001 & 5 & 2 & 0.002 & $-0.001^{*}$ \\
\hline 5 & 1 & 0.001 & 5 & 3 & 0.000 & $0.001^{*}$ \\
\hline 5 & 1 & 0.001 & 5 & 4 & 0.000 & $0.001^{*}$ \\
\hline 5 & 2 & 0.002 & 5 & 3 & 0.000 & $0.002^{*}$ \\
\hline 5 & 2 & 0.002 & 5 & 4 & 0.000 & $0.002^{*}$ \\
\hline
\end{tabular}

Note: *significant at $\alpha=.05$ level. Family-Wise Error (FWE) was separately controlled at each level of perturbation at which simple main effect tests were performed. 


\section{ET AL}

For the three-way interaction effect between $\mathrm{FO} \times \mathrm{PL} \times \mathrm{RS}$ on the dependent variable: Localmax, the interaction effect of PL $\times$ RS was studied at each level of FO, and the results are presented only graphically in Figures 7 through 9. The three figures show clearly that for all FO levels, Localmax values were close between RS levels 2, 3 and 4 across all PL levels. At level 1 of FO, level 1 of RS diverged from the other RS levels in Localmax values at and above level 3 of PL, at level 2 of FO clear discrepancy started to occur at level 4 of PL, and at level 3 of FO a very large difference was observed between level 1 of RS and the other RS levels at the highest PL level.

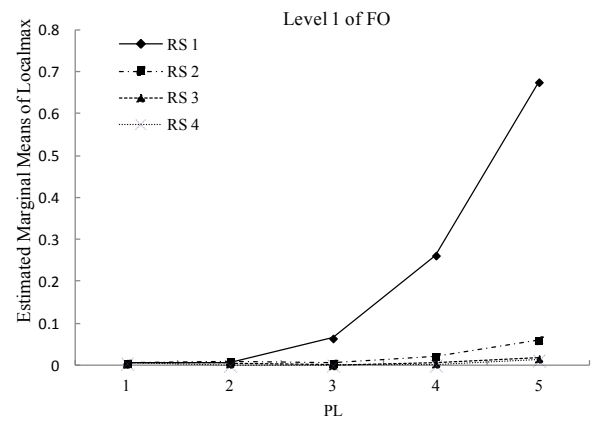

Figure 7. PL $\times$ RS for Localmax at level 1 of $\mathrm{FO}$

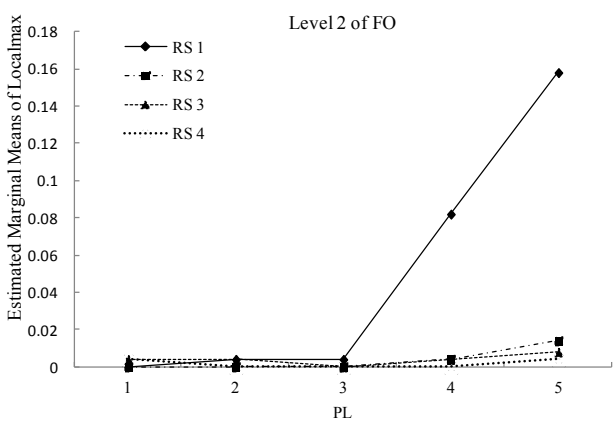

Figure 8. $\mathrm{PL} \times \mathrm{RS}$ for Localmax at level 2 of $\mathrm{FO}$

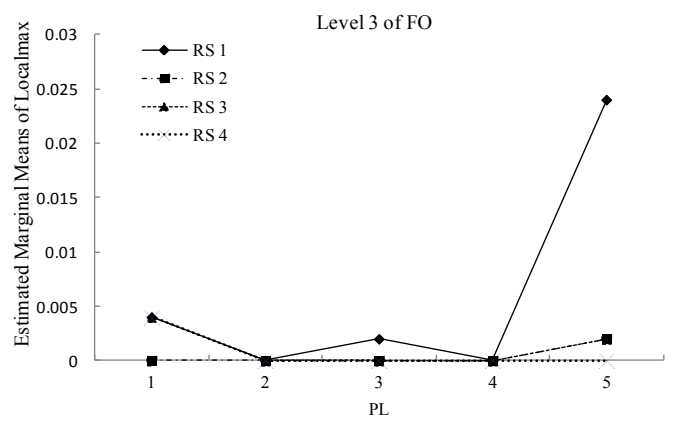

Figure 9. $\mathrm{PL} \times \mathrm{RS}$ for LogMatch at level 3 of $\mathrm{FO}$ 


\section{MPLUS IN GMM ESTIMATION}

\section{Conclusions}

Results from the factorial ANOVA analyses showed that in both simulation studies MCONV was not a significant factor affecting convergent solutions in Mplus, nor was SVQ studied in Simulation 1. The results related to SVQ were different from those findings obtained by Jones and Nagin (2007) who found that using informed starting values from the means of the growth parameters coming from a latent class growth analysis helps avoid the occurrence of local maxima solutions.

In terms of the main effects, RS and FO showed the same patterns of outcome means obtained under the two simulation studies. As was expected, low Dlog, high Logmatch, and low Localmax mean values were all associated with high levels of RS, indicating higher RS should be used to increase the likelihood of proper convergence of growth mixture model parameters. The story of the main effect for FO, though, was more complex and somewhat confusing. Both simulation studies showed that as levels of FO increased, the Dlog mean values became smaller (which is a desirable outcome) while the Localmax mean values increased (which is an undesirable outcome). Although Simulation 1 also showed an increased Logmatch mean value with increased levels of FO, the choice of FO still needs to be carefully considered. This is because the impacts of using various levels of FO are not consistent in terms of their impact on the four desirable properties of convergence that were considered in this study.

In terms of the main effect for PL, both simulation studies indicated that the largest Localmax value and the lowest Logmatch were found with the highest PL level considered in the study, suggesting that a high PL would not be a desirable choice for obtaining proper convergence solutions. In fact, results from Simulation 1 showed that a moderate PL level (e.g., level 3) was favored for the lowest Localmax outcome. Among all the significant factors, MC was the only factor in Simulation 1 that affected all four outcome variables and explained most of the variance in Negvariance. As was expected, when the investigated model was very complex, proper convergence solutions were negatively impacted. At its highest level, the 4-class model had the highest Dlog mean value, the lowest Logmatch mean value, the largest Negvariance mean value, and the highest Localmax mean value, all of which suggest unstable convergence solutions.

Though an assessment of main effects provides a general idea of the marginal effects of the manipulated factors, a more complete understanding of the influence of these factors on convergence to proper solutions may be obtained by assessing the presence and nature of interaction effects among these independent 


\section{ET AL}

variables. The only two-way interaction effect $(\mathrm{PL} \times \mathrm{MC})$ identified in Simulation 1 showed that the highest Dlog value was with the highest level of MC across all PL levels and that Dlog values showed the slowest increase for level 1 of MC starting from level 3 of PL.

The interaction between PL and MC also affected Localmax. The highest Localmax mean values always occurred with the most complex model across levels of PL. The observation that the lowest Localmax mean values were found at (a) level 1 and 2 of MC at PL level 3 and (b) at level 3 of MC at PL level 2 suggests that a high PL was not a desirable choice for obtaining a low Localmax outcome. The assessed interaction effect between PL and FO found in Simulation 2 resulted in the following findings with respect to PL: (a) Dlog values increased for all levels of FO as PL increased, (b) Logmatch mean values started to decrease in magnitude for all levels of FO at level 4 of PL, and (c) Localmax values began to increase at or above level 4 of PL for all levels of FO. Either the increase or the decrease was wanted, which suggests that a high PL level (e.g., level 4 or 5) was not a desirable choice for obtaining effective convergence of parameter estimates.

A similar complex and confusing story with respect to the choice of a desirable level of FO occurred as what was found for the main effect of FO. As FO increased, Dlog values increased for each PL level, Lower Logmatch values were always found with higher FO for higher levels of PL, but lower Localmax values were found with higher FO for higher levels of PL. These findings indicate a conflicting situation where the choice of FO was especially challenging when a higher PL level was used.

Study of the two-way interaction effect of PL $\times$ RS on Logmatch and Localmax showed most substantial mean differences occurred at higher levels of PL (e.g., level 4 and 5 of PL) where level 4 of RS was found having the highest Logmatch value and the lowest Localmax value, suggesting that a high RS be considered when high PL has to be used. Also, considering the decrease of Logmatch value and the increase of Localmax value for all RS levels occurred obviously at and above level 4 of PL, it was also suggested that higher PL levels not be used. The interaction effect between RS and FO for the dependent variable Localmax showed no significant mean differences between levels of FO across RS levels 2 through 4 . This finding suggests that the choice of FO should not be a big concern when RS is large.

The three-way interaction effect $(\mathrm{MC} \times \mathrm{PL} \times \mathrm{RS})$ for the dependent variable Nonconverge found in Simulation 1 showed no significant findings at level 1 and level 2 of MC, suggesting that with a less complex model (i.e., the 2 and 3-class models), the choice of levels of PL and RS would not significantly affect 


\section{MPLUS IN GMM ESTIMATION}

convergence solutions. At level 3 of $\mathrm{MC}$, significant Nonconverge mean differences were found only at level 5 of PL, with RS level 3 and 4 (having the lowest Nonconverge value) showing no significant mean difference. This finding shows with very complex models, high RS should be used, especially when the PL level is very high. The three-way interaction effect between FO, PL and RS obtained with Localmax showed somewhat similar patterns for the two-way interactions between PL and RS for each of the three FO conditions. At middle and lower levels of PL, Localmax values were similar in magnitude for all levels of RS and remained stable. When PL level was very high, Localmax values at level 1 of RS not only increased sharply but deviated dramatically from the Localmax values at the other RS levels.

\section{Recommendations}

Based on the findings and conclusions previously discussed, some recommendations that users of Mplus may wish to follow when fitting GMMs are as follows:

- Use a large number of random start values, especially when the perturbation level is high.

- Number of random starts should be at least at level 2 for lower number of local maxima when the perturbation level is high. However, the number of random starts does not greatly affect the number of non-converged solutions if moderate perturbation levels are used.

- $\quad$ Choice of the number of final optimizations needs to be considered carefully. Usually, a high number of final optimizations is not recommended although the choice would not be a big concern when the number of random starts is high.

- Do not use high perturbation levels (e.g., level 4 or level 5), especially with very complex growth mixture models which may show much higher rate of increase in number of local maximum solutions than less complex models. Instead, a moderate perturbation level (e.g., Mplus default perturbation level of 5) is recommended for obtaining better convergence solutions.

- With less complex growth mixture models, the choice of number of random starts and perturbation levels may not be so important. However, when a model is very complex (e.g., the 4-class model), a 


\section{ET AL}

high number of random starts should be considered for obtaining better convergence solutions, especially when perturbation level is very high.

\section{References}

Bauer, D. J. (2007). 2004 Cattell award address: Observations on the use of growth mixture models in psychological research. Multivariate Behavioral Research, 42: 757-786.

Böhning, D. (1999). Computer-assisted analysis of mixtures and applications: Metaanalysis, disease mapping and others. Monographs on statistics and applied probability. New York: Chapman \& Hall/CRC.

Cohen, J. (1988). Statistical power analysis for the behavioral sciences (2nd ed.). Hillsdale, NJ: Erlbaum.

Dempster, A. P., Laird, N. M, \& Rubin, D. B. (1977). Maximum likelihood from incomplete data via the EM algorithm. Journal of the Royal Statistical Society. Series B (Methodological), 39: 1-38.

Hamilton, J. (2009). An investigation of growth mixture models when data are collected with unequal selection probabilities: A Monte Carlo study (Unpublished doctoral dissertation). University of Maryland, College Park.

Harring, J. R. (2012). Finite mixtures of nonlinear mixed effects models. In J. R. Harring \& G. R. Hancock (Eds.), Advances in longitudinal methods in the social and behavioral sciences. Charlotte, NC: Information Age Publishing, Inc.

Hipp, J. R., \& Bauer, D. J. (2006). Local solutions in the estimation of growth mixture models. Psychological Methods, 11: 36-53.

Hsu, J. C. (2011). Estimation and model selection for finite mixtures of latent interaction models (Unpublished doctoral dissertation). University of Maryland, College Park.

Jennrich, R. I., \& Schluchter, M. D. (1986). Unbalanced repeated-measures models with structured covariance matrices. Biometrics, 42: 805-820.

Jones, B. L., \& Nagin, D. S. (2007). Advances in group-based trajectory modeling and a SAS procedure for estimating them. Sociological Methods \& Research, 35: 542-571.

Kohli, N. (2010). Estimating unknown knots in piecewise linear-linear latent growth mixture models (Unpublished doctoral dissertation). University of Maryland, College Park. 


\section{MPLUS IN GMM ESTIMATION}

Liu, J., \& Harring, J. R. (2012). A systematic investigation of within-subject and between-subject covariance structures in growth mixture models (Unpublished doctoral dissertation). University of Maryland, College Park.

McLachlan, G. J., \& Basford, K. E. (1988). Mixture models: Inference and applications to clustering. New York: Marcel Dekker.

McLachlan, G. J., \& Krishnan, T. (2008). The EM algorithm and extensions. New York: Wiley.

McLachlan, G. J., \& Peel, D. (2000). Finite mixture models. New York: Wiley.

Meredith, W., \& Tisak, J. (1990). Latent curve analysis. Psychometrika, 55: 107-122.

Muthén, B. (2001). Latent variable mixture modeling. In G. A. Marcoulides $\&$ R. E. Schumacker (Eds.), New developments and techniques in structural equation modeling (pp. 1-33). Mahwah, NJ: Erlbaum.

Muthén, B. O. (2004). Latent variable analysis: Growth mixture modeling and related techniques for longitudinal data. In D. Kaplan (Ed.), Handbook of quantitative methodology for the social sciences (pp. 345-368). Newbury Park, CA: Sage Publications.

Muthén, B. O., \& Shedden, K. (1999). Finite mixture modeling with mixture outcomes using the EM algorithm. Biometrics, 55: 463-469.

Muthén, L., \& Muthén, B. O. (2010). Mplus user's guide (6th ed.). Los Angeles: Muthén \& Muthén.

Nagin, D. S. (1999). Analyzing developmental trajectories: A semiparametric, group-based approach. Psychological Methods, 4: 139-177.

Tolvanen, A. (2008). Latent growth mixture modeling: A simulation study (Unpublished doctoral dissertation). University of Jyvaskyla.

Wang, M., \& Bodner, T. E. (2007). Growth mixture modeling: Identifying and predicting unobserved subpopulations with longitudinal data. Organizational Research Methods, 10: 635-656. 


\section{ET AL}

\section{Appendix}

For the simulation, the number of occasions of measurement were fixed at $p=6$. The covariance structures were constructed to be constant across groups and are defined as:

$$
\operatorname{cov}(\boldsymbol{\eta})=\Psi=\left(\begin{array}{cc}
1 & \\
.224 & 2
\end{array}\right) \quad \operatorname{cov}(\boldsymbol{\varepsilon})=\Theta=\sigma^{2} \mathbf{I}, \text { where } \sigma^{2}=.75
$$

The mean vectors for the three-class model are specified as

$$
\begin{aligned}
& \alpha_{1}^{\prime}=(4.5,-.85) \\
& \alpha_{2}^{\prime}=(2.5,-.05) \\
& \alpha_{3}^{\prime}=(1.4,-.70)
\end{aligned}
$$

Data were generated according to the two-stage approach outlined by Hipp and Bauer (2006). 